\title{
Mesh Migration into Sigmoid Colon after Inguinal Hernioplasty Presenting as Lower Abdominal Mass: A Case Report
}

Hamza Ahmad ${ }^{1 *}$, Haroon Javaid Majid ${ }^{2}$, Ayesha Shahid ${ }^{1}$

${ }^{1}$ Surgical Trainee Resident, Shaikh Zayed Hospital, Lahore, Pakistan

${ }^{2}$ HOD General Surgery 1, Shaikh Zayed Hospital, Lahore, Pakistan

Correspondence to: Hamza Ahmad, Surgical Trainee Resident, Shaikh Zayed Hospital, Lahore, Pakistan. Received date: July 21, 2021; Accepted date: July 29, 2021; Published date: August 5, 2021

Citation: Ahmad H, Majid HJ, Shahid A (2021) Mesh Migration into Sigmoid Colon after Inguinal Hernioplasty Presenting as Lower Abdominal Mass: A Case Report. J Med Res Surg 2(4): pp. 1-3. doi: 10.52916/jmrs214052

Copyright: (C2021 Ahmad H, et al. This is an open-access article distributed under the terms of the Creative Commons Attribution License, which permits unrestricted use, distribution and reproduction in any medium, provided the original author and source are credited.

\section{ABSTRACT}

Mesh related visceral complications are, in general, considered to be infrequent and rarely reported in literature, but in the last decade, incidence of such complications have increased. Our study highlights the case of an elderly patient, who developed rare complication of Mesh migration after laparoscopic inguinal hernioplasty.

\section{Keywords:}

Hernioplasty, Herniorrhaphy, Hernia, Colonoscopy, Enterocutaneous Fistula, Tenesmus

\section{Introduction}

Hernioplasty has documented advantages over herniorrhaphy, which caused increased use of Mesh in hernia repair. Complications of hernioplasty are usually minor but Mesh related visceral complications have been reported in literature [1]. Mesh can migrate into viseras like bladder [2], and intestines presenting as obstruction [3] or enterocutaneous fistula [4] or even mass [5]. Our report presents a rare case where diagnosis of diverticulosis with a sigmoid mass was made but, to our shock, intraoperatively Mesh migration was noted.

\section{Case Report}

A 71 year old person presented in Out Patient Department (OPD) with off and on lower abdominal pain for 1 year, along with a history of tenesmus, now progressively increasing for the last 7 days. Pain was moderate, dull, non-radiating and relieved after defecation. Patient underwent laparoscopic inguinal hernioplasty 9 years back. Upon examination, the patient was vitally stable with mild tenderness at left iliac fossa with a $5 \times 4 \mathrm{~cm}$ smooth, tender mass, non adherent to skin, was palpable. Digital Rectal Exam (DRE) was unremarkable. Patient's baseline investigations were within normal range. Patient stool examination showed occult blood, with serum Carcinoembryonic Antigen (CEA) and Cancer (CA) 19-9 levels within normal range. Colonoscopy showed multiple diverticula in sigmoid and descending colon with a bezoar impacted in sigmoid colon. Attempts to remove it remained unsuccessful. Differentials of colonic diverticulosis,impacted bezoar and sigmoid mass were made. Computed Tomography (CT) scan reported extensive large bowel diverticulosis along sigmoid and descending colon with a thickening of proximal sigmoid colon. A thick walled collection extending from the proximal sigmoid loop anteriorly along the posterior reflection of anterior abdominal wall, extending posteriorly to left rectus abdominis reaching to supra pubic level (Figure 1). This has a frank communication with bowel lumen. Previous hernia repair noted with multiple metallic anchoring sutures in situ (Arrow in
Figure 2), at least two are within the large collection (Arrow in Figure 3). Underlying colonic tumors can not be excluded. There was no pelvic or intra abdominal lymphadenopathy noted.
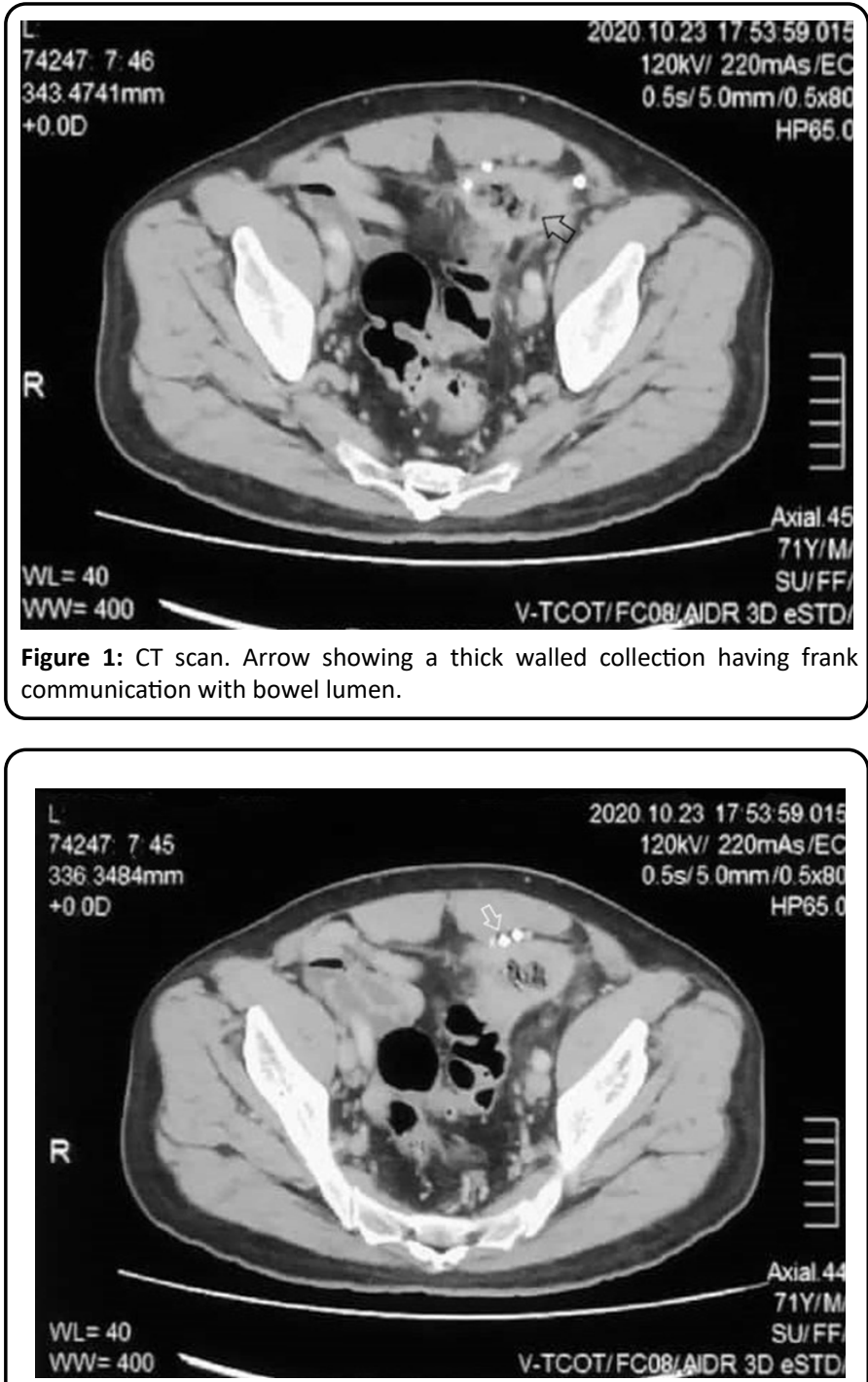

Figure 2: CT scan. Arrow showing metallic anchoring suture of previous hernial repair. 


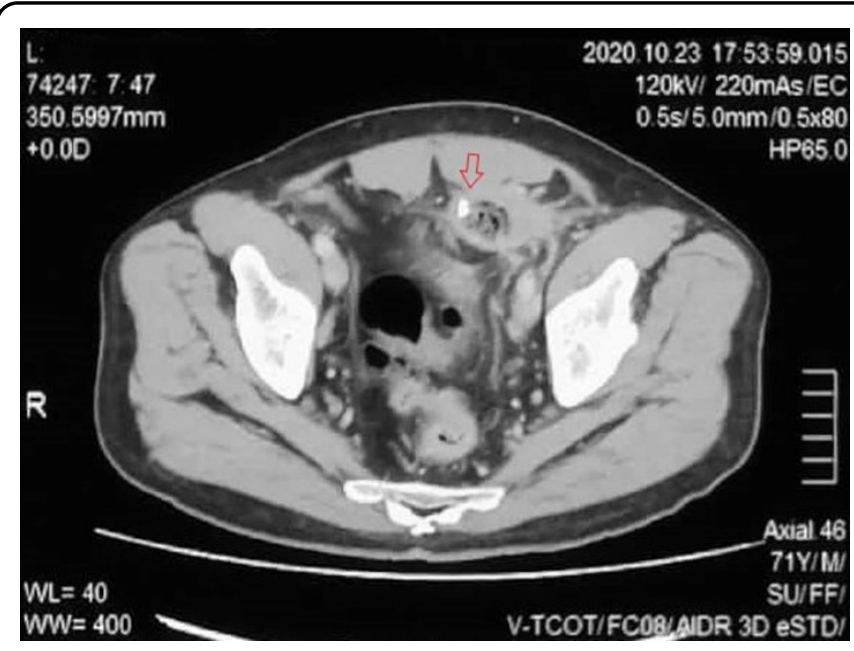

Figure 3: CT scan. Arrow showing at least two metallic anchoring suture with in large collection.

An exploratory laparotomy was planned to remove this above mentioned collection with possibly colonic repair or colectomy. Patient was optimized for surgery and surgery was done in November, 2020. After inducing general anesthesia Bilateral ureteral catheters were passed via cystoscope to identify ureters during surgery. A midline incision was made and rectus sheath was dissected along with peritoneum,descending colon along with sigmoid colon was identified. A feculent smell was noticed. A black mass was identified eroding sigmoid colon (Figure 4-5), along with multiple diverticula present in sigmoid colon. Mass was carefully removed and identified as Mesh from previous surgery, and sent for culture sensitivity. Thickening of proximal sigmoid colon was noted, which was removed via mini resection of sigmoid colon and end to end anastomosis was done. Defect of mysentry was also stitched up and peritoneal cavity was washed with warm saline and abdomen was stitched up. Post operative period was unremarkable. Patient was discharged on 9th POD on oral medication. Patient is asymptomatic till this day.

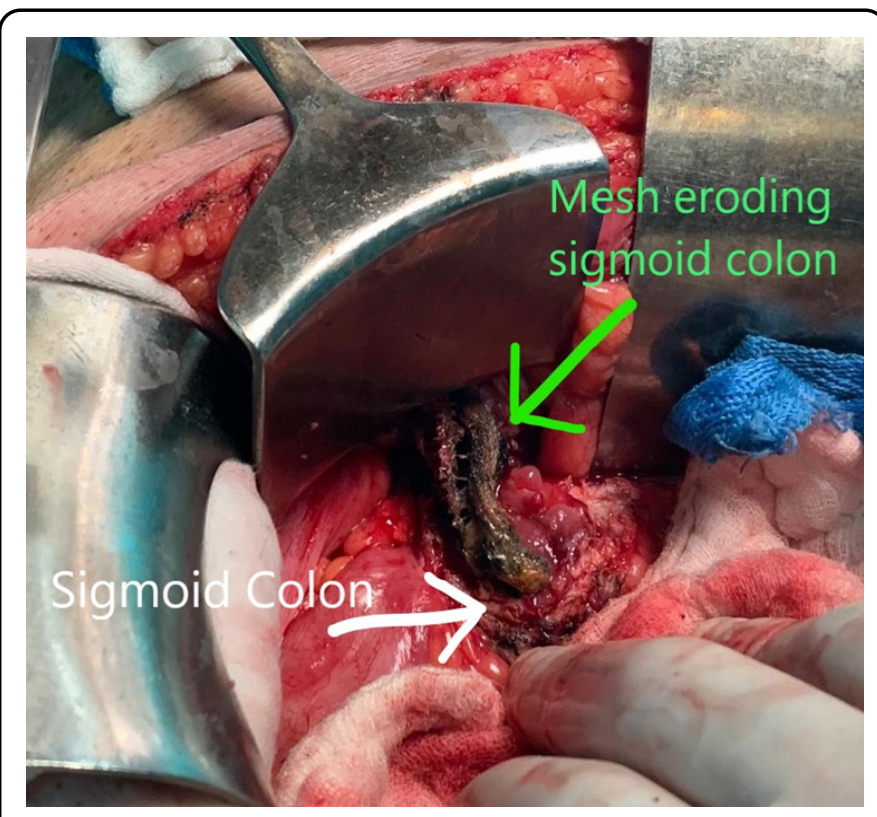

Figure 4: Intraoperative picture showing mesh eroding sigmoid colon.

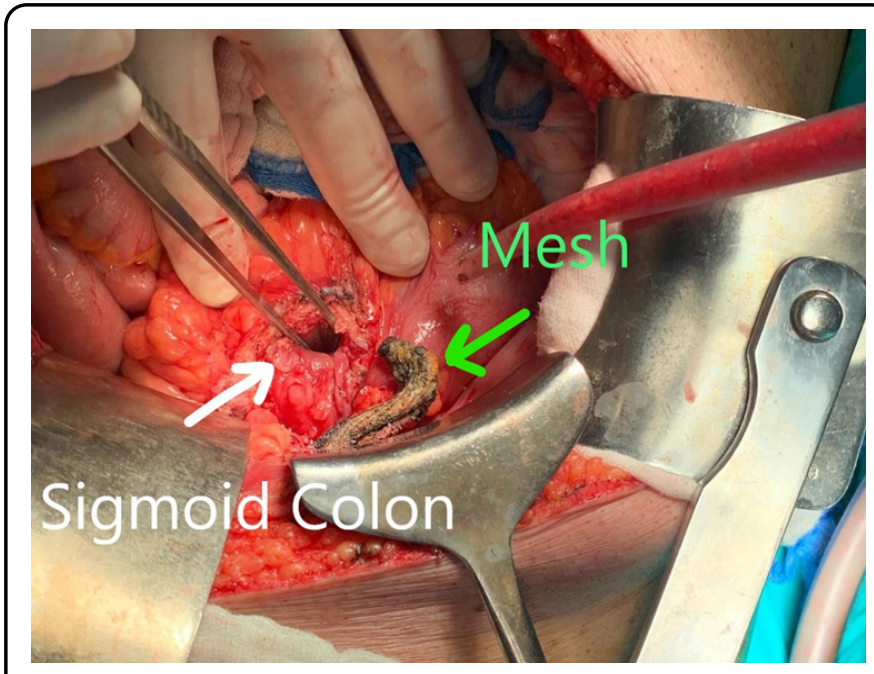

Figure 5: Intraoperative picture showing mesh removed from sigmoid colon.

\section{Discussion}

Hernioplasty through laparoscopic approach is not exclusive of complications. Though, overall complication index of laparoscopic procedure is less than open procedures [6]. Complications after laparoscopic hernioplasty are usually minor i.e. seroma, hematoma, orchitis etc. Complication due to Mesh like foreign body reaction, deeply seated infections andMesh migration into viscras leading to perforation have been reported occasionally [7]. The intestine and urinary bladder are among the commonest, involved in Mesh migration. A relatively rare case of Mesh migration mimicking as colon polyp has been reported previously [8].

Depending upon the relationship of migrating Mesh with visceral organs, manifestation of clinical symptoms may vary and present after one to 20 years after hernioplasty [8]. In the majority of cases patients were presented with lower abdominal pain and mild tenderness, while incidence of bowel obstruction, palpable mass, weight loss and anorexia was reported by few reports $[5,9]$. In our case the patient was presented with lower abdominal pain with tenesmus and no other significant positive signs which led to delay in diagnosis. Typical signs of perforation and peritonitis were absent, though formation of diverticula made the diagnosis of Mesh migration ambiguous. Colonoscopy identified a bezoar in sigmoid colon, but due to rarity of Mesh migration, it was overlooked as a possible diagnosis. Every symptom was pointing towards diverticulosis, but thick walled collections raised the suspicion of lesion. Tumor markers came out to be within normal range. A plan of exploration was made. Other studies have shown that migrating Mesh are usually misdiagnosed as intra abdominal mass [10] or even neoplasm [5], based on radiological examination.

Inadequate fixation, incomplete peritoneal repair or improper amount of space for implantation of Mesh are important factors for Mesh migration into visceral organs [11]. Which in turn can lead to obstruction as well as enterocutaneous fistulas. Furthermore tackers, used to fix Mesh in laparoscopic hernioplasty and sharp edges of Mesh can cause erosion through intra abdominal inflammatory processes [12]. As our patient was mostly asymptomatic, factors leading to painless Mesh migration can be these. 
1. Foreigh body reactions to Mesh lead to chronic inflammation which in turn lead migration of Mesh through anatomical planes, gradually [12].

2. Bacterial biofilm can be formed over migrating Mesh due to chronic contamination with gram positive or negative cocci, aiding in painless migration. (In our case, Mesh has growth of E. Coli which are known to form multispecies biofilm [13].

3. Foreign body (In our case prosthetic Mesh) reduces formation of mesothelial layer of peritoneum, causing scarring, which can lead to localized inflammation.

\section{Conclusion}

Once erosion of Mesh is established, for possible prevention of further erosion, and preservation of affected viscera, partial or complete resection is preferred [14]. To prevent long term Mesh related complications, tailoring of Mesh as well suture placement should be appropriate. Like any other surgery, sticking to principles of antisepsis should be strictly followed to reduce long term complications of hernioplasty.

Conflict of Interest: The authors do not have any conflict of interest to declare.

\section{Funding: None.}

\section{References}

1. Gossetti F, D'Amore L, Annesi E, et al. (2019) Mesh-related visceral complications following inguinal hernia repair: an emerging topic. Hernia 23(4): pp. 699-708.

2. Asano H, Yajima S, Hosoi $Y$, et al. (2017) Mesh penetrating the cecum and bladder following inguinal hernia surgery: a case report. J Med Case Rep 11: p. 260.

3. Manzini G, Henne-Bruns D, Kremer M (2019) Severe complications after mesh migration following abdominal hernial repair: report of two cases and review of literature. GMS Interdiscip Plast Reconstr Surg DGPW 8.
4. Bostanci O, Idiz UO, Yazar M, et al. (2015) A Rare Complication of Composite Dual Mesh: Migration and Enterocutaneous Fistula Formation. Case Rep Surg 2015: 293659.

5. Asano H, Yajima S, Hosoi Y, et al. (2017) 13 Mesh penetrating the cecum and bladder following inguinal hernia surgery: a case report. J Med Case Rep 11(1): p. 260.

6. Millikan KW, Kosik ML, Doolas A (1994) A prospective comparison of transabdominal preperitoneal laparoscopic hernia repair versus traditional open hernia repair in a university setting. Surg Laparosc Endosc 4(4): pp. 247-253.

7. Wegener ME, Chung D, Crans C, et al. (1993) Small bowel obstruction secondary to incarcerated Richter's hernia from laparoscopic hernia repair. J Laparoendosc Surg 3(2): pp. 173176.

8. Liu S, Zhou XX, Li L, et al. (2018) Mesh migration into the sigmoid colon after inguinal hernia repair presenting as a colonic polyp: A case report and review of literature. World J Clin Cases 6(12): pp. 564-569.

9. El Hakam MZ, Sharara Al, Chedid V (2010) Persistent left lower abdominal pain. Gastroenterol 138: pp. e5-e6.

10. Ojo P, Abenthroth A, Fiedler P, et al. (2006) Migrating mesh mimicking colonic malignancy. Am Surg 72(12): pp. 1210-1211.

11. Demir U, Mihmanli M, Coskun H, et al. (2005) Comparison of prosthetic materials in incisional hernia repair. Surg Today 35(3): pp. 223-227.

12. Agrawal A, Avill R (2006) Mesh migration following repair of inguinal hernia: a case report and review of literature. Hernia 10(1): pp. 79-82.

13. Beloin C, Roux A, Ghigo JM (2008) Escherichia coli biofilms. Curr Top Microbiol Immunol 322: pp. 249-289.

14. Lauwers P, Bracke B, Hubens G, et al. (2003) Unusual complications of preperitoneal mesh implantation in the treatment of inguinal hernia. Acta Chir Belg 103(5): pp.513-516. 